\title{
Tsafon
}

Revue d'études juives du Nord

$75 \mid 2018$

Exil des langues - Langues d'exil

\section{Exil : le cas des poètes yiddish américains}

\section{Marie Schumacher-Brunhes}

\section{(2) OpenEdition}

Journals

Édition électronique

URL : https://journals.openedition.org/tsafon/553

DOI : $10.4000 /$ tsafon.553

ISSN : 2609-6420

\section{Éditeur}

Association Jean-Marie Delmaire

\section{Édition imprimée}

Date de publication : 1 juin 2018

Pagination : 15-34

ISSN : 1149-6630

\section{Référence électronique}

Marie Schumacher-Brunhes, «Exil : le cas des poètes yiddish américains », Tsafon [En ligne], 75 | 2018,

mis en ligne le 06 mai 2019, consulté le 23 juin 2021. URL : http://journals.openedition.org/tsafon/553 ; DOI : https://doi.org/10.4000/tsafon.553 


\title{
Exil : le cas des poètes yiddish américains
}

\author{
Marie Schumacher-Brunhes*
}

Le yiddish, langue en perpétuel exil ${ }^{1}$ qui s'est forgée au rythme des migrations d'une partie du peuple juif, est souvent présenté comme le paradigme culturel de l'exil historique qui a mené les Juifs ashkénazes de l'Europe de l'Ouest vers l'Europe de l'Est puis vers l'Amérique. L'émigration en Amérique a tout d'une «expérimentation radicale » ${ }^{2}$ pour reprendre le mot de Pierre Anctil, parce qu'il s'agit, au départ, d'un continent vierge de toute influence judaïque et, par la suite, parce qu'elle peut être vue comme un condensé des processus d'émancipation, qui s'accompagnent possiblement d'un sentiment de perte et de déréliction : la séparation physique d'avec le Vieux Monde tend à générer une remise en question des liens anciens. Si l'on y regarde bien, nombre des écrivains yiddish qui viennent en Amérique du Nord partagent les aspirations de leurs contemporains et homologues du camp hébraïque qui gagnent la Palestine ; l'élan est similaire. Et de fait, l'Amérique est bien ce lieu unique où pourront se succéder trois générations d'écrivains yiddishophones.

La frontière entre exil et émigration est cependant ténue ${ }^{3}$. Outre les circonstances qui président au départ, c'est probablement aussi une affaire de perception individuelle et collective, laquelle dépend à la fois

\footnotetext{
* Université de Lille, SHS.

${ }^{1}$ Le yiddish, «langue de l'exil», comme le qualifia Isaac Bashevis Singer dans le discours prononcé pour la réception de son prix Nobel de littérature en 1978.

2 Pierre Anctil, «Vers une relecture de l'héritage yiddish montréalais », Études françaises, vol. 37, n³, 2001, p. 23.

3 Pour faire le point sur ces notions d'exil, d'émigration, de déplacement, de déracinement ou encore de nostalgie, on pourra consulter l'article de Ségolène Payan, « Du déplacement au sentiment d'exil », Recherches en psychanalyse, vol. 1, n9, 2010, p. 171-182. Elle définit le sentiment d'exil comme un «échec du déplacement », p. 176.
} 
de l'intensité de l'attachement culturel à ce qu'on laisse derrière soi et de l'horizon d'attente vis-à-vis du pays que l'on gagne. Un autre paramètre consiste bien entendu dans la possibilité, théorique ou pratique, du retour. Celle-ci existe globalement pour les Juifs jusque dans les années trente, bien que de manière plus ténue durant la Première Guerre mondiale, et se répercute de façon remarquable dans la perception que les poètes ont d'eux-mêmes. Ainsi, progressivement, les écrivains yiddish découvrent la vulnérabilité de la langue dans laquelle ils créent ou ont choisi de créer. Beaucoup ont le sentiment d'être les représentants d'une littérature de transition soumise aux pressions de l'acculturation et de l'individualisme propres à la société immigrante.

Nous nous proposons ici de cheminer avec les trois générations d'écrivains évoquées plus haut: les poètes prolétariens d'abord, suivis par les représentants du mouvement Di Yunge [Les Jeunes], qui leur succèdent dans la première décennie $d u X X^{\mathrm{e}}$ siècle en se détournant ostensiblement d'eux au nom de l'Art pour l'art, et enfin, autour de la revue Inzikh [En soi] fondée en 1920, les Introspectionnistes, qui, instruits aux États-Unis, lecteurs de Freud et fins connaisseurs de la poésie anglophone, mettent au défi les précédents. Nous nous pencherons alors sur le recueil In land... fun mayn gebeyn [Au pays de mes os] ${ }^{4}$ de la poétesse Kadia Molodowsky (1874-1975) venue tardivement de Varsovie, en 1935, sur le territoire américain. Tout au long de cette œuvre publiée en 1937, la poétesse, arrivée en Amérique « saturée d'exils différents ${ }^{5}$ comme elle le soulignera en 1972 dans les passages de son autobiographie consacrés à la gestation de ce recueil, se confronte avec ses propres exils au rebours de l'esprit conquérant de ses prédécesseurs (ce qui n'exclut pas le scepticisme chez ces derniers) pour conclure au caractère incurable de ce sentiment.

\section{Di goldene medine ? Les poètes face à l'Amérique}

Les écoles poétiques modernistes - Yunge puis Inzikhistn - vont s'attacher à s'affranchir du référent de l'immigration/émigration exploré dans les premières années du $\mathrm{XX}^{\mathrm{e}}$ siècle par les poètes prolétariens ${ }^{6}$. La

\footnotetext{
${ }^{4}$ Kadye Molodowski, In land... fun mayn gebeyn, Chicago, Farlag Shteyn, 1937.

5 Kadye Molodowski, Fun mayn elterzeydns yerushe, chap. 52-54, Svive, n³7, september 1972, p. 52-63, ici p. 61-62.

${ }^{6}$ Voir par exemple Marc Miller, Representing the Immigrant experience. Morris Rosenfeld and the Emergence of Yiddish Literature in America, Syracuse, NY, Syracuse University Press, 2007.
} 
poésie d'un Yoysef Bovshover, d'un David Edelshtat ou encore d'un Morris Vinshevsky rendait compte en effet, sur un ton généralement offensif, des phénomènes d'exploitation et d'aliénation indissociables de l'expérience du migrant.

Parmi ces poètes, Morris Rosenfeld est celui qui, chroniquant le quotidien des tenements [immeubles d'habitation disposant généralement d'un rez commercial des métropoles américaines] et des ouvriers des ateliers de confection dans les sweatshops [ateliers où l'on exploite le personnel], articule le mieux ce triple bannissement des masses urbaines exilées de chez elles, coupées de la nature, et par extension, exilées de $\mathrm{Dieu}^{7}$, même si l'on peut considérer à bien des égards que le shtetl a été transplanté en Amérique ${ }^{8}$.

Son évocation des conditions de vie misérables des ouvriers, de la voracité des patrons, de la dureté des métiers revient à user du Moi poétique, comme le ferait tout poète romantique (sans ambition esthétique toutefois), pour évoquer une expérience que partage tout amant, tout ouvrier, tout père de famille s'inquiétant pour sa fille aînée ou constatant avec amertume qu'il ne voit sa progéniture qu'endormie. Le sentiment d'exil ne trouve que rarement à s'exprimer dans la poésie de Rosenfeld, que submerge une imagerie parfois revendicative puis de plus en plus sentimentale, voire larmoyante, confirmant au fond le caractère définitif de l'installation des Juifs en Amérique. Ainsi, le poème «A goles marsh» [Une marche de l'exil], qui sera mis en musique par H. Gershowitz, fait-il plutôt figure d'exception. Surtout, il s'inscrit dans la tradition de l'expression de la condition exilique du Juif, qui est d'abord un exil spirituel, et de l'aspiration qui la sous-tend à réunir le corps et l'esprit du peuple juif en Terre d'Israël dans l'attente des temps messianiques :

Le bâton du marcheur en main / Sans foyer ni pays / Sans main secourable, sans ami / Sans présent, sans lendemain / Nulle part toléré, mais de partout chassé / Au-delà de la nuit ne restant séjourner / Toujours souffrir, souffrir, souffrir /

\footnotetext{
${ }^{7}$ Nous reprenons ici la définition que donne de son œuvre David G. Roskies. Voir David G. Roskies, "Coney Island, USA: America in the Yiddish Literary Imagination ", The Cambridge Companion to Jewish American Literature, ed. Hana Wirth-Nesher, Michael P. Kramer, Cambridge University Press, 2003, p. 72.

8 Cf. Gerald Worin, Tradition Transformed: the Jewish Experience in America, Baltimore, J. Hopkins UP, 1997. Voir notamment le chapitre «Transplanted in America : Smaller Cities and Towns », p. 91-106.
} 
Toujours courir, courir, courir / Toujours plus vite, toujours plus loin, allonger le pas / Tant que vos forces ne vous abandonnent pas. ${ }^{9}$

Les poètes prolétariens, qui portent ce nom parce qu'ils partagent entièrement le quotidien misérable des premiers immigrants (ainsi Dovid Edelshtat, qui meurt de la tuberculose à 26 ans), exprimaient dans leur œuvre un peu brusque, didactique ou sentimentale sur le mode du lamento, une solidarité de classe qui résistait à l'idée qu'un talent puisse se développer individuellement. Les années qui précèdent le déclenchement de la Première Guerre mondiale voient en revanche se multiplier des œuvres littéraires qui, bien que portées par des auteurs partageant pour la plupart l'idéologie socialiste de leurs prédécesseurs, abandonnent le terrain de la lutte sociale pour se confronter individuellement à la promesse de l'américanisation : ainsi le roman In keslgrub [Dans le tourbillon $]^{10}$ de David Ignatov, paru sous forme de feuilleton entre 1907 et 1912, ou encore la nouvelle de Lamed Shapiro intitulée Oyfn Yam [Sur la mer] ${ }^{11}$. Dans ce dernier texte, le narrateur s'applique, au fil des pages rythmant la traversée de l'Atlantique, à se positionner par rapport à l'effort d'acculturation qu'exige l'Amérique de ses immigrants. La restitution de ces tâtonnements et de ces interrogations, et donc l'accentuation du Je qui en découle, reflète une rupture progressive avec la culture traditionnelle - qui vue de l'extérieur apparaît comme une communauté monolithique, presque indissoluble - et donc avec tous les marqueurs d'identité qui y sont associés tel l'exil. Libéré des définitions qui lui étaient accolées jusqu'alors, le Je peut révéler tout son potentiel créatif.

Les Yunge forment dès 1907 le premier groupe d'avant-garde de la littérature yiddish, avant même que ne se constituent les groupes

\footnotetext{
${ }^{9}$ Morris Rosenfeld, «A goles marsh », Shriftn, vol. 1, New York, Evalenko, 1908, p. 100. Nous n'avons reproduit ici que le premier couplet. Notre traduction comme pour toutes les autres citations. « Mit dem vandershtab in hant,/ On a heym un on a land/ On a goyel on a fraynd,/ On a morgn on a haynt/ Nit geduldet nor geyogt/ Vu genekhtikt nit getogt/ Immer vey, vey, vey/ Immer gey, gey, gey/ Immer shpan, shpan, shpan/ Kol zman koyakh iz faran ».

${ }^{10}$ David Ignatoff, In keslgrub, New York, Inzl, 1918. D. Ignatoff désigne ainsi la ville de New York.

${ }^{11}$ Lamed Shapiro, "Oyfn yam », Di yidishe melukhe un andere zakhn, New York, Naytsayt, 1919, p. 193-229. "Oyfn yam » est paru une première fois à Varsovie en 1910. On pourra se reporter à l'analyse de Leah Garrett, " Shipping the Self to America: The Perils of Assimilation in Glatshteyn's and Shapiro's Immigration Novels », Melus, 2001, vol. 26, n³, p. 203-229, ici notamment, p. 208-214.
} 
expressionnistes et futuristes de Pologne ou de Russie. Ils se trouvent à l'intersection entre le symbolisme du XIX ${ }^{\mathrm{e}}$ siècle finissant, notamment sa déclinaison russe, et l'imagisme anglo-américain. Cette nouvelle génération de poètes yiddishophones est en quête d'un langage pur, débarrassé des tics du langage parlé ou de la langue littéraire observables alors (germanismes répandus dans la presse et chez les poètes prolétariens, hébraïsmes du monde traditionnel), lavé de tout sentimentalisme, de toute emphase. Leur chant du cygne est le recueil Indzl [île] paru en 1925-1926. Leur ambition de poètes alchimistes est de surpasser la poésie des poètes prolétariens en mettant fin à la complicité historique entre les écrivains et la presse yiddishophone. Le dynamisme de la vie culturelle yiddish d'alors n'est pas le moindre des atouts des Jeunes : la presse est florissante (le Forverts réalise le plus gros tirage parmi la presse des minorités linguistiques), le théâtre s'épanouit comme il n'est jamais parvenu à le faire en Europe, les suppléments littéraires se multiplient, lancés par des rédacteurs en chef socialistes ouverts à toutes les nouvelles idées.

C'est dans cette ambiance que naît leur mouvement dont la seule allégeance est à la littérature et qui identifie dans la presse yiddishophone l'ennemi à combattre. Pour donner corps à leurs idées, ces écrivains lancent, à l'hiver 1907-1908, l'éphémère revue Di Yugnt. Dans leur manifeste programmatique, ils se fixent pour objectif de «créer pour la littérature yiddish en Amérique une maison bien à elle, un foyer indépendant qui la libèrera de l'exil dans lequel on la tient enfermée $»^{12}$. Il faut entendre ici dans le mot « exil» l'abandon des Belles lettres aux mains de la presse. On entend là toute la bravoure des pionniers, au mépris de leur inexpérience et des affres de la pauvreté dans lesquelles tous se débattent. Leurs noms sont Mani Leib ${ }^{13}$, Reuvn Eyzland, Zishe Landoy, Moyshe Leyb Halpern, Dovid Ignatov, ils sont bottier, serveur, laveur de vitres, chapelier, livreur de journaux, triment tout le jour afin de pouvoir se retrouver la nuit, à moins qu'ils ne se fassent porter pâles pour retrouver leurs comparses dans la cantine lactée de Goodman and

\footnotetext{
${ }^{12}$ Éditorial non signé mais dont $\mathrm{D}$. Ignatov s'est réclamé plus tard être l'auteur. Voir $D i$ Yugend, $\mathrm{n}^{\circ} 2$ (janvier 1908), Nyu york, Progresiv poblishing assn, 1-2. Le numéro 2 porte la mention « a tsaytshrift vos hot di oyfgabe tsu zamlen in zikh di shafungen fun bloyz yunge shrayber » [Une revue qui se donne pour objectif de rassembler la création d'écrivains dont le seul signe distinctif est d'être jeunes].

${ }^{13}$ Dans un poème autobiographique tardif publié à l'automne 1932, dans Di tsukunft, et intitulé « Ikh bin », Mani Leib déclare : « Je ne suis pas, grâce à Dieu, un cordonnier qui écrit / Mais un poète qui fabrique des chaussures ».
} 
Levine's ou chez Sholem's. Leur zèle ne diffère guère de celui des Juifs religieux sur le Vieux continent; ils s'autoqualifient d'ailleurs de «minyan d'un genre nouveau ${ }^{14}$. Tous sont convaincus d'avoir mis un terme à leur exil et, tenants de l'Art pour l'art, consacrent toute leur énergie à donner forme à leurs idéaux esthétiques et artistiques. Indubitablement, la libération des valeurs esthétiques qu'ils prônent se fait dans le langage de la liberté américaine. Chacun puise dans ce nouveau terreau, chacun y sème, et la moisson est d'une rafraîchissante hétérogénéité en dépit de la similitude des situations de départ. Au cœur de beaucoup d'œuvres, on trouve la ville de New York et son maelstrom cosmopolite $^{15}$. La solitude qu'elle induit favorise la prise de conscience de sa propre individualité. Outre le roman d'Ignatov In keslgrub évoqué plus haut, citons ici le recueil de nouvelles New Yorkish de Lamed Shapiro, ou encore le roman que Joseph Opatoshu consacre en 1913 aux bas-fonds new-yorkais, Moris un zayn zun Filip, écrit dans un style presque naturaliste anticipant la scission du mouvement qui se produira l'année suivante.

Dans ce paysage, Moyshe Leyb Halpern (1886-1932) occupe une place tout à fait singulière. Né en Galicie orientale, envoyé de son Zlotshev natal comme apprenti à Vienne, il arrive en Amérique en 1908 et fait le pari de l'excentricité et de l'indépendance financière, refusant tout travail alimentaire, toute compromission, et cultivant à l'envi son image de " poète maudit». Lorsque le groupe des Yunge se scinde en 1914, il ne prend parti ni pour les uns ni pour les autres et publie des parodies des deux camps. Il produit une poésie pastorale des poubelles, des cordes à linge, des rares parcs du Lower East Side où trois arbustes se disputent un coin de ciel. Son autre lieu de prédilection est le rivage de Coney Island, ce lieu qui marque, selon David G. Roskies, l'extrême frontière de l'exil ${ }^{16}$. Ses premiers poèmes sont rassemblés en 1919 et paraissent dans un recueil intitulé, sans surprise, In New York (1919) où

\footnotetext{
${ }^{14}$ Comme le rappelle le critique Shmuel Niger faisant le point en 1918 sur la nouvelle génération de poètes yiddish américains. Shmuel Niger, "Yung Amerike », Onheyb, $\mathrm{n}^{\circ} 5$, April 1918. Signalé par Ruth Wisse, A Little Love in Big Manhattan - Two Yiddish Poets, Cambridge, Harvard University Press, 1988, p. 4.

${ }^{15}$ Il faudrait inclure ici la rencontre avec les Noirs américains (pas toujours exempte de stéréotypes mais généralement empathique) telle qu'elle se déploie dans de nombreux poèmes (cf. la quarantaine d'entre eux repris par exemple dans l'anthologie de N. Mayzil, Amerike in yidishn vort) ou encore dans le recueil de poèmes en prose de Y. Y. Shvarts Kentoki [Kentucky].

${ }^{16}$ David G. Roskies, «Coney Island, USA... », op.cit., p. 70-91.
} 
se croisent ivrognes, femmes abandonnées ou solitaires et où résonne le yiddish de la rue. Loin d'être le poète moderniste enfermé dans sa tour d'ivoire, Halpern défend « un modernisme qui vient d'en bas ${ }^{17}$. La figure du joueur de tambour ambulant, qui donne son nom au poème de 1911 «Der Gasnpoyker » ${ }^{18}$ symbolise cette destitution économique, la confusion psychique qui en découle, l'absence d'utilité sociale. Plus personne n'écoute le tambour dont la peau tendue finit par éclater sous les coups de baguette acharnés. Pour autant, le résultat de cet isolement du poète n'est ni la colère ni le désespoir, simplement une forme de fébrilité, la prolifération d'images métaphoriques. Halpern ne parviendra jamais à articuler une vision collective d'harmonie, ce à quoi s'attache précisément Mani Leib.

Cinq ans plus tard paraît un deuxième recueil, Di goldene pave [Le Paon d'or] ${ }^{19}$, en référence au motif folklorique qui veut que le paon d'or de la chanson d'amour symbolise le messager porteur de mauvaises nouvelles. Ce titre évocateur cache en fait une épopée ironique sur l'exil, " une ontologie de la condition de sans-foyer ${ }^{20}$, selon les termes de Ruth Wisse, dans laquelle le paon d'or devient le symbole de la perte et des désillusions, le déplacement du Juif, de l'immigrant, du poète, dévoilant au fil des vers "la dislocation de l'homme dans l'univers ${ }^{21}$. Chez Halpern, la ville moderne est omniprésente, mais dans une représentation en forme de palimpseste, et non pas strictement allégorique : en permanence, des choix lexicaux convoquent la mémoire des paysages de l'Europe de l'Est, proposant ainsi au lecteur un autre cadre de référence et la possibilité d'un refuge. C'est particulièrement frappant à la lecture d'un poème qu'il intitule, en auto-référence à un poème portant le même titre et paru cinq ans plus tôt, "In Sobvey » [Dans le subway $]^{22}$. L'ambition n'est plus, cette fois, de restituer les sons, les secousses, les odeurs du métro, grand topos de la littérature juive américaine. Au lieu de cela, le voyage en métro donne lieu à des bouffées

\footnotetext{
${ }^{17}$ Julian Levinson, « Modernism from Below : Moyshe Leyb Halpern and the Situation of Yiddish Poetry », Jewish Social Studies, vol. 10, 2004, p. 148.

${ }^{18}$ Moyshe Leyb Halpern, In nyu-york, New York, Farlag Vinkel, 1919. Le poème est paru initialement en 1911 dans le journal satirique Der kibitser.

${ }^{19}$ Moyshe Leyb Halpern, Di goldene pave, Cleveland, Farlag grupe yidish, 1924.

${ }^{20}$ R. Wisse, A Little Love..., op. cit., p. 136 : « an ontology of homelessness ».

${ }^{21}$ Ibid.

${ }^{22}$ M. L. Halpern, Di goldene pave, op. cit., p. 87. On pourra se reporter ici à l'analyse qu'en donne Julian Levinson, "On Some Motifs in Moyshe Leyb Halpern: A Benjaminian Meditation on Yiddish Modernism », Prooftexts, vol. 32, n 1, 2012, p. 7781.
} 
mémorielles qui viennent recouvrir l'expérience immédiate. Le poète regagne son domicile au petit matin. Son attention se concentre sur un passager assis en face de lui, un asiatique reconnaissable à ses yeux bridés et sa carnation :

Moi, fatigué d'une nuit à boire du vin/ Et à regarder les jolies filles / Je rentre chez moi. / Et lui...il part fournir au monde le fruit de son turbin. / On se dévisage, / Moi - les yeux ensommeillés derrière mes lunettes / Et lui - avec ces deux sortes de fentes qu'il a dans le visage, / Qui font ressurgir en moi l'image de bains, de bains réservés aux femmes / Où jadis / En cachette (par un tout petit trou) / J'ai collé mon œil et regardé. ${ }^{23}$

Dans les yeux impassibles de ce voyageur qui soulignent la dissolution de toute solidarité de classe tandis que la déchéance du poète rentrant d'une nuit de débauche est prononcée, le poète découvre subitement un élément qui le ramène au Vieux Monde. Les yeux du Chinois réduits à deux fentes font émerger soudain dans sa mémoire la fente dans la cloison du bain rituel, à travers laquelle le jeune homme, des années plus tôt, observait les femmes à la dérobée. Figurativement, les yeux du Chinois sont une porte qui ouvre sur le passé. Le métro, lancé dans sa course, l'emmène en fait dans un voyage dans le temps. De même, pour décrire les secousses qu'imprime le métro aux voyageurs, Halpern aura recours au verbe «shoklen», par ailleurs utilisé pour désigner le mouvement de balancement des Juifs religieux lorsqu'ils prient ou étudient, superposant ainsi à la scène profane du voyage en métro l'image d'une scène empreinte de religiosité. On pourrait, dans cette volonté d'Halpern d'ouvrir des espaces pour la mémoire du Vieux Monde, être tenté de lire la possibilité d'une réconciliation entre le Juif et le moderniste citadin. Mais ce serait oublier des choix lexicaux qui ne laissent que peu de doutes sur les intentions du poète, ainsi le choix du slavisme "pratse » pour évoquer le turbin dont la sonorité fait immédiatement ressurgir les ambiances sonores de jadis ou celui du mot sécularisé « bod » qu'il préfère au vocable d'origine hébraïque « mikve », inscrit lui dans la tradition judaïque, pour désigner le bain rituel. Ainsi, le télescopage des deux visions vise bien plutôt à générer le grotesque.

\footnotetext{
${ }^{23} \mathrm{Ibid}$. « Ikh a mider fun a nakht mit vayn /un vaybersheynkeyt / for aheym. / Un er... fort di velt bazorgen mit zayn pratse. / Kukn mir zikh on, / Ikh - mit oygn shloffarkholemt durkh briln, / Un er - mit shpares tsvey azoy in zayn ponim, / vos moln oys far mir a bod a vayberish, / Vuhin ikh hob a mol / Farganevet (durkh a lekhl bloyz) / Arayngekukt».
} 
Le recueil se referme sur le cycle «Zarkhi», sorte de double nocturne du poète. Dans ce cycle, Halpern, se moquant de tout ce qui est sacralisé par les autres, détruit le concept même de Sehnsucht, en en mettant en évidence l'absurdité : ce qui est de l'autre côté de l'océan est au moins aussi hideux, pour ne pas dire davantage, que ce qui se trouve sur la rive américaine, du côté du poète, telle la fille en chaleur guère appétante du poème "Zarkhi à lui-même". La mort elle-même, qui rattrape Zarkhi, n'apporte ni répit, ni consolation, comme l'indique la parodie du Memento mori de Halpern lui servant d'épitaphe ${ }^{24}$.

\section{Le retour du refoulé}

En dépit de la fascination qu'exerce sur eux l'Amérique, en dépit de leur volonté crânement affirmée de se distinguer de ce qu'ils appellent "la yiddishkeyt du tsholent ${ }^{25}$ (en référence au plat traditionnel du shabbat), en dépit encore du parricide symbolique auquel s'apparente le numéro d'hommage édité à la mort du «père de la littérature yiddish moderne », l'écrivain varsovien Y. L. Peretz, Eisland y réfutant toute influence littéraire ${ }^{26}$, il serait cependant vain de croire que «di alte heym » a disparu de l'horizon de ces poètes. Ce serait en effet perdre de vue une réalité essentielle: ces artistes vivent pleinement entre deux mondes, ils ont le regard en permanence tourné vers l'Europe, dans une relation qui est, selon les termes de l'analyse d'Avrom Novershtern, violemment œdipienne, faite de concurrence et d'exploitation réciproque ${ }^{27}$.

On échouera à définir des tendances car pour un témoignage de nostalgie à l'égard du Vieux Monde, il se trouvera trois exemples de rejet et de rébellion, et chaque ode à l'Amérique peut être démontée par une critique tout aussi hyperbolique. Cette grande variété de rapports traduit

\footnotetext{
${ }^{24}$ Voir D. G. Roskies, « Coney Island... », op. cit., p. 82.

${ }^{25}$ R. Wisse, A little love..., op. cit., p. 60.

${ }^{26}$ Mais ce même Eisland claque la porte de la revue Literatur un lebn après avoir découvert l'insolente recension que donne Zishe Laudau du numéro d'hommage à Peretz, " l'homme de lettres varsovien qui vient de nous quitter».

${ }^{27}$ David Roskies, "Here Dwells the Jewish people », In geveb: a Journal of Yiddish Studies, August 2015, p. 3.

Consultable en ligne à l'adresse : https://ingeveb.org/articles/review-here-dwells-thejewish-people (consulté le 2 mars 2018). Cet article est une recension de l'ouvrage paru en hébreu de Avraham Novershtern, Here Dwells the Jewish People: A Century of American Yiddish Literature, Jerusalem, The Magnes Press, 2015. Le titre choisi par Noversthern fait référence au poème de H. Leivick : « Do voynt dos yidishe folk » qui fait du Lower East Side new-yorkais une terre d'élection pour les Juifs.
} 
en réalité une relation spirituelle très forte entre les deux rives de l'océan. Il en résulte que lorsque ces écrivains et intellectuels pensent à l'Europe, ce n'est, a priori, pas un mouvement rétrospectif empli de nostalgie, mais bien quelque chose de prospectif qui naît de l'échange permanent avec les maisons d'édition, les cercles et les cénacles, les bibliothèques, les revues et la presse yiddish. Ces échanges s'établissent dans une relation de donnant-donnant d'autant plus fertile que beaucoup de ce qui constituait une entrave à la publication (la censure par exemple) disparaît avec la renaissance de la Pologne au lendemain de la Première Guerre mondiale et les possibilités offertes tout un temps aux écrivains soviétiques. L'Entre-Deux-Guerres, en dépit de l'assimilation d'une partie de la population yiddishophone via notamment la scolarisation dans les écoles publiques polonaises et soviétiques, constitue, faut-il le rappeler, l'époque la plus riche sur le plan de la création en yiddish.

On ne saurait pourtant ignorer le tournant que constitue le déclenchement de la Première Guerre mondiale. Le conflit, avec ses nouvelles inquiétantes, la nouvelle vague d'immigration qu'elle génère, provoque chez beaucoup un regain d'intérêt pour le passé. Souvent, plus le séjour américain des écrivains yiddish se prolonge, plus s'affirme dans leur œuvre le retour aux paysages et aux realia du Vieux Monde. Au point que la trajectoire artistique des Yunge, loin d'être celle d'une acculturation progressive, s'apparente à un processus d'aliénation par rapport à la culture américaine qui avait pu susciter chez eux tant d'enthousiasme et dont ils se détachent ou se détournent désormais, sans que ce geste, nous l'avons dit, soit nécessairement teinté de nostalgie. Les voyages qu'entreprennent H. Leivick, Joseph Opatoshu, Jacob Glatstein, Baruch Glasman, pour ne citer que ceux-là, en URSS en sont un signe, quand bien même la lecture idéologique en demeure évidente. Le statut d'exilés que les auteurs yiddishophones américains rejetaient fièrement à leur arrivée, parce que le déplacement ne pouvait être un échec, finit donc par transparaitre dans leur production ${ }^{28}$. Cette tendance est plus que perceptible dès la fin de la Grande Guerre, dans la salve de ballades de Mani Leib (pas moins de trois recueils publiés en l'espace de douze mois en 1918) ${ }^{29}$, dans les poèmes convoquant le shtetl de l'enfance

\footnotetext{
${ }^{28}$ Voir Ruth Wisse, « Di Yunge: Immigrants or Exiles? », Prooftexts, n¹, 1981, p. 4361.

${ }^{29}$ Mani Leib publie successivement à la maison Inzl les recueils Lider, dans lequel il magnifie la composante germanique du yiddish, Baladn, où il met à l'honneur la
} 
(ainsi l'élégie de R. Eisland sur sa ville natale de Tarnów), ou encore dans le roman historique In poylishe velder [Dans les forêts de Pologne] de Y. Opatoshu. Plus qu'une nostalgie pour di alte heym, il faut lire dans ces textes le souci d'établir fermement une sorte de mythe des origines pour poètes déracinés. Rétroactivement, face aux horreurs traversées par les communautés juives d'Europe centrale et orientale, leur départ quelques années plus tôt se charge chez certains de culpabilité et d'un sentiment de perte irrémédiable.

Cette évolution s'accompagne d'une prise de conscience douloureuse: le nombre de yiddishophones sur le sol américain commence à s'amenuiser, l'attachement au yiddish apparaissant comme rétrograde à ceux qui réussissent et l'apport de sources vives se tarissant sous la pression nativiste. Au mieux, les romans-feuilletons et le cinéma entretiennent-ils une nostalgie de bon aloi. Progressivement, le centre de la création yiddish retrouve son berceau européen. Les écrivains qui ont lié leur destin au yiddish voient donc leur lectorat se détourner d'eux. Quelle peut donc encore être la pertinence d'une telle écriture ? Par contraste, le Vieux Monde, rempli de matrones, de bouchers rituels, de rabbins thaumaturges apparait d'une infinie robustesse. L'inspiration de Mani Leib, qui est devenu le poète phare des anthologies et des manuels scolaires des écoles yiddish, s'essouffle. Sa tentative de rejoindre les rangs de la poésie engagée en initiant une collaboration avec le journal communiste Di Frayheyt en 1926 provoque la crise la plus profonde dans la vie du poète : l'année suivante, il cesse d'écrire.

\section{Les derniers héritiers}

Pour comprendre la poésie que déploie le groupe des Introspectivistes (ou encore Introspectionnistes) autour de la revue Inzikh, dont le premier numéro paraît à New York en janvier 1920, et de l'anthologie In $z i k h^{30}$, publiées toutes deux par Yankev Glatstein, Aaron Glants-Leyeles et Nokhem Borekh Minkov, il faut envisager cette dernière dans une double perspective : ces poèmes en effet, bien que rédigés en yiddish et destinés par là-même au seul lectorat

composante hébraïque, puis Yidishe un slavishe Motivn, ode au yiddish ukrainien de son enfance.

${ }^{30}$ In zikh: a zamlung introspektive lider, New York, M. N. Mayzel Farlag, 1920. Le volume s'ouvre sur le manifeste de leur mouvement, intitulé "Instropektivizm » et déjà publié dans le premier numéro de la revue (p. 5-27). Les poètes s'y désignent comme « introspektivistn » mais le terme «inzikhistn » va s'imposer. 
yiddishophone, sont cartographiés simultanément dans les espaces yiddish et américain. Leurs auteurs, bien que fidèles à leur langue maternelle, rejettent l'exil et s'appliquent à creuser leur sillon sur le sol américain, offrant une poésie qui, pour reprendre l'image utilisée par Joseph Landis pour décrire la production de ces poètes évoluant essentiellement à New York, «germe de l'asphalte américain » ${ }^{31}$. La recension que le poète Mikhl Likht donne en 1921 du recueil Chicago Poems (1916) du poète américain d'origine suédoise Carl Sandburg permet de comprendre ce qui anime ces jeunes gens :

C'est pour nous que Sandburg découvre l'Amérique; il est la meilleure définition de l'Amérique pour nous. Les gens qu'il côtoie, l'air qu'il respire, ses fleurs, sa Pocahontas SONT notre atmosphère... Parce qu'après tout, nous sommes l'Amérique et qu'elle nous est chère. ${ }^{32}$

Ainsi, ces poètes yiddish, par leur identification avec l'Amérique, participent fondamentalement de l'environnement américain qui les englobe, qu'il s'agisse de leur environnement direct, celui de la grande ville, ou des grands espaces et des mythes qui y sont attachés, les montagnes, le Far West, les Indiens. Ce faisant, ils prennent congé de la " goldene medine », le pays doré chanté et espéré par les immigrants juifs fuyant l'Europe centrale et orientale.

Cette rupture avec la rhétorique qui a longtemps prévalu découle d'une nouvelle manière d'appréhender l'Amérique qu'expose Nokhem Minkov en 1922 dans sa Lettre new-yorkaise publiée dans le troisième numéro de la deuxième série de Inzikh : l'Amérique est " une chose ", non pas une croyance, un style, une conception. Cette réité permet aux poètes immigrés écrivant en yiddish d'y participer, d'apporter, au sens propre du terme, leur pierre à l'édifice, de « faire » l'Amérique ${ }^{33}$. Ce qui ne veut pas dire pour autant qu'ils s'auto-identifient comme Américains. La poésie nourrie d'Amérique qu'ils écrivent en yiddish se pare à leurs yeux d'authenticité, devient une part authentique de cette réalité. Par conséquent, ce qui constitue par excellence l'une des clés de la poésie

\footnotetext{
31 Joseph Landis, "Yiddish dreams in America », Handbook of American-Jewish Literature: An Analytic Guide to Topic, Themes and Sources, Westport, CT, Greenwood Press, 1988, p. 143-165, ici, p. 146.

32 Cité par Merle Bachman, Recovering "Yiddishland»: Threshold Moments in American Literature, Syracuse University Press, 2008, p. 210. Les italiques et majuscules sont de Likht. Notre traduction de l'américain.

${ }^{33}$ Ibid, p. 211. Pour l'original, voir Nokhem Minkov, « New Yorker briv », Inzikh 2, n³, déc. 1922, p. 76-78.
} 
moderniste, le thème de l'aliénation du poète, notamment dans la rencontre avec la grande ville, ne se retrouve pas nécessairement chez eux. Au contraire, la foule anonyme des métropoles, le chaos, la fragmentation, la rudesse des atmosphères urbaines sont vécus comme autant de promesses de renaissance et de libération.

L'ambition de repousser les frontières du yiddish, affichée par ces poètes dans leur manifeste, ne trouve que peu d'écho au sein de la communauté yiddishophone. Cette dernière ne s'identifie guère avec leur enthousiasme pour les possibilités insoupçonnées de la langue et, partant, leur manière de se saisir de la « chose américaine » avec d'autant plus de facilité qu'ils maîtrisent pour la plupart parfaitement l'anglais. " Nous sommes, disent-ils, des 'poètes juifs' parce que nous sommes juifs et écrivons en yiddish. [...] Mais le poète ne saurait se mettre en quête de cette judéité et en faire montre $»^{34}$. Leur décision de vocaliser les mots d'origine hébraïque et d'entériner ainsi l'autonomisation du yiddish par rapport à son ascendance (l'allemand et l'hébreu) résulte de cette prise de position, qui les écarte encore plus du lecteur yiddishophone sans pour autant les rapprocher du lecteur américain. Lancés dans cette surenchère, tant par rapport à leurs collègues américains que par rapport à la poésie moderniste " internationale », ils parviennent à une véritable alchimie formelle, créant ainsi de façon effectivement «miraculeuse » ${ }^{35}$ une littérature qui fait entrer sur un mode virtuose la poésie yiddish dans la littérature universelle ${ }^{36}$.

Le drame de ces auteurs est que la communauté potentielle représentée par les écrivains américains, et, par-delà, le lectorat américain, ne soupçonne pas même leur existence. Souvent, les auteurs yiddish sont versés dans la langue et la littérature de la culture majoritaire, mais l'inverse n'est jamais vrai. C'est là une situation sur laquelle revient constamment Reuben Eisland dans ses mémoires

\footnotetext{
34 «Introspektivizm», In zikh, op. cit., p. 19. « Mir zenen 'yidishe poetn' dermit, vos mir zenen yidn un shraybn yidish. [...] Es iz nit di oyfgab fun dem dikhter aleyn tsu zukhn un aroysvayzn zayn yidishkeyt $\gg$.

${ }^{35}$ Selon le mot même de leur chef de file. Voir Yankev Glatshteyn, « Yidishe literatur in Amerike, a nes ?», In tokh genumen, vol. 2, New York, Matones, 1947. «Nes» signifie « miracle » en hébreu.

${ }^{36}$ Pour une analyse poussée de cette poésie, on pourra se reporter au chapitre «Une littérature ancienne-nouvelle » de l'essai de Carole Ksiazenicer-Matheron intitulé «Dans la pliure du temps: littérature yiddish et modernités (quelques parcours d'écriture) » consultable à l'adresse :

http://www.vox-poetica.org/sflgc/biblio/matheron.html [consulté le 2 mars 2018].
} 
intitulés Fun undzer friling [Notre printemps] ${ }^{37}$, dont la première partie se concentre sur la période 1905-1915, le «printemps » des poètes et écrivains juifs américains. S'il convoque dans la courte préface «les arômes âcres du bourgeonnement, la palette de couleurs de la floraison, l'agitation, les tourments, les rêves, les aspirations, les attentes, l'insolente assurance propre aux jeunes pousses que l'on trouve dans tous les printemps ${ }^{38}$, il évoque au fil des pages qui suivent la quête inlassable de mécènes et de financements, les multiples tentatives de mettre en place des structures, telles des sociétés bilingues d'auteurs, qui permettraient une telle reconnaissance, mais aussi l'infatigable activité de traduction à laquelle se prêtent ces écrivains et dans laquelle il faut voir un autre geste d'enracinement sur le sol américain. L'intérêt persistant et profond dont font montre les poètes yiddish à l'égard de la poésie américaine, Whitman en tête, mais aussi du folklore amérindien (par l'intermédiaire des traductions anglaises qui en sont données, notamment dans la revue Poetry) ${ }^{39}$ doit en effet être compris comme un acte d'interprétation et de transformation destiné à asseoir simultanément le modernisme et l'américanisme du poète yiddish immigrant; en d'autres termes, à naturaliser ce dernier. Cet intérêt pour la création coterritoriale était palpable dès le premier numéro de la revue Shriftn (1912) dans lequel Y. Y. Shvarts présentait sa traduction de «Salut au monde » de Whitman. Il se confirme dans les discussions, les recensions et les traductions de poésie américaine qui émaillent les séries successives de Inzikh.

\section{Saturée d'exils : la poétesse Kadia Molodowsky}

Kadia Molodowsky, née en 1894 en Biélorussie, que le projet sioniste ne tente guère au début de sa vie, est profondément attachée à la vie en diaspora. Bien qu'il soit volontaire, elle vit comme un exil son départ de Varsovie pour New York en 1935 dans un contexte

\footnotetext{
${ }^{37}$ Ruvn Ayzland, Fun undzer friling - literarishe zikhroynes un portretn, aroysgegebn fun Ruvn Ayzland -Yublkomitet Miami Beach, New York, Farlag Inzl, 1954.

${ }^{38}$ «mit di harbe reykhes fun oyfshprots, ale kolirn fun bli, dem umru, di veytikn, di khaloymes, di benkshaftn, die dervartungen un di hoferdikeyt fun yungn vuks, vos shpirt zikh in ale frilingen ».

39 Rachel Rubenstein consacre plusieurs pages à cet intérêt des Yunge pour le primitivisme, qui s'inscrit clairement dans une démarche moderniste, en se demandant toutefois s'il ne vise pas plutôt les poètes de la revue Poetry que la culture amérindienne elle-même. Voir son livre Members of the Tribe: Native Americans in the Jewish Imagination, Detroit, Wayne State University Press, 2010, p. 64 sqq.
} 
mouvementé ${ }^{40}$. Lorsqu'elle reprend la plume sur le continent américain, elle le fait dans une grande solitude, coupée à la fois de ses lecteurs européens mais aussi de ses homologues américains, dont le lectorat va s'amenuisant au fur et à mesure de l'américanisation.

Chez elle, l'exil est double : il est géographique mais il s'agit aussi d'un exil au sein-même de la culture interne du judaïsme traditionnel, laquelle cantonne la femme à une culture de la dévotion se déployant exclusivement en yiddish. La poétesse d'expression yiddish définit en premier lieu sa poésie "contre» la culture attitrée des femmes, « en exil », comme le dirait Kathryn Hellerstein, « dans sa propre langue ${ }^{41}$. Ce second exil, Kadia Molodowsky l'embrasse sur fond d'un parcours personnel atypique pour sa génération : son père, en effet, a veillé à ce qu'elle reçoive une éducation religieuse en hébreu, ce qui est assez exceptionnel, et sa maîtrise de la langue lui permet de l'enseigner en plus de son activité au sein des écoles yiddish du réseau TSYSHO (Di Tsentrale Yidishe Shul-Organizatsye). Ne comptant guère sur ses pairs en dépit du programme ambitieux que se sont fixé les avant-gardes yiddish, force est de constater que les poétesses juives demeurent rejetées à la marge - Kadia Molodowsky s'interroge d'emblée sur la question cruciale de savoir comment une poétesse juive yiddishophone peut réconcilier son art avec la définition que le judaïsme traditionnel donne du rôle de la femme et par là-même redonner une cohérence à la langue qu'à certains égards elle transgresse. Tous les poèmes, romans, nouvelles, essais, pièces de théâtre, textes autobiographiques et articles journalistiques publiés entre 1927, date de la parution de son premier recueil de poésie Kheshvendike Nekht [Nuits d'automne] aux prestigieuses éditions Kletskin, et sa mort en 1975, constituent une

\footnotetext{
${ }^{40}$ Elle accepte l'invitation du directeur de la maison d'édition new-yorkaise Matones, sachant qu'une partie de sa famille s'est installée à Philadelphie. Auparavant, K. Molodowky aura croisé le fer avec pratiquement tout le monde : avec le célèbre Melech Ravitch pour son article condescendant paru dans Literarishe bleter sur la production des poétesses d'expression yiddish (1927), avec la presse varsovienne qui assure la prolifération du shund (1933), ou encore avec les leaders du Bund qu'elle accuse de corruption dans leurs accords avec le réseau scolaire TSYSHO (1934-1935).

${ }^{41}$ Cette partie s'appuie très largement sur les travaux difficilement égalables de Kathryn Hellerstein, notamment ses traductions de la poésie de Kadia Molodowsky, Paper Bridges, Selected Poems of Kadya Molodowsky, trad., éd. et intr. Kathryn Hellerstein, Wayne University Press, 1999, ainsi que le long article «In Exile in the Mother Tongue: Yiddish and the Woman Poet », Borders, Boundaries and Frames: Cultural Criticism and Cultural Studies - Essays from the English Institute, ed. Mae G. Henderson, New York, Routledge, 1995, p. 64-106.
} 
tentative de réponse à cette question lancinante, doublée de questionnements concernant la survie des Juifs dans le monde moderne.

Chez de nombreux auteurs, on constate que les conflits intérieurs liés à l'exil géographique peuvent être résolus en retrouvant une sérénité intérieure : l'écrivain, l'artiste, compense le déracinement en faisant de son intériorité son nouveau foyer. Plus graves sont les exils intérieurs ou spirituels face à une adversité, ceux qui ébranlent l'âme. Le drame de Kadia Molodowsky réside en cela qu'elle n'est jamais vraiment parvenue à reprendre pied dans sa langue : le sentiment de déracinement, de perte irrémédiable liée à l'arrachement au vivier linguistique et culturel qui est le sien (au sens large, car Kadia Molodowsky était une grande voyageuse en Europe orientale; elle ne se fond pas avec un lieu, une ville particulière) l'ébranle si profondément que la poésie elle-même, dans cette phase de sa création, ne parvient pas à devenir le refuge qu'elle a pu constituer pour tant de poètes et d'écrivains. L'exil géographique se double donc d'un exil spirituel (autre que celui, ontologique, du Juif religieux) qui provoque une véritable dislocation de l'âme. C'est précisément parce qu'elle s'est extraite du monde traditionnel, qu'elle s'est détachée de la tradition en faisant le choix du sécularisme et de la modernité, que l'ébranlement que ressent Kadia Molodowsky à son départ d'Europe pour les USA est si profond : le départ de l'Europe pour les États-Unis, aussi douloureux soit-il, n'aurait sinon été pour elle qu'un simple déplacement d'un point à l'autre de l'exil, de l'exil compris comme diaspora - goles.

C'est son cinquième recueil de poésie, qu'elle publie quelques mois après son arrivée aux États-Unis, qui constituera le cœur de la présente réflexion. Ce recueil au titre déchirant In land... fun mayn gebeyn [Dans le pays de mes os] paru en 1937 constitue en effet une internalisation de l'exil, déployée dans une ligne fragmentée de poèmes que rien ou peu ne lie ensemble. Suite à la publication de ce recueil, elle réduira considérablement son activité d'écriture, même si on ne peut parler de silence puisqu'elle maintient une activité éditoriale et publie un roman autobiographique Fun Lublin Biz Nyu-York: Togbukh fun Rivke Zilberg [De Lublin à New York : Journal de Rivke Zilberg] (1942). Elle retrouve définitivement une voix avec la publication en 1946 du recueil Der Melekh Dovid Aleyn Iz Geblibn [Seul le Roi David a subsisté] qui contient un nombre important de khurbn-lider, ces poèmes qui thématisent l'Holocauste. Elle s'installe un temps en Israël, au tournant 
de la décennie 1950, avant de revenir aux États-Unis. Son dernier ouvrage est une autobiographie en prose dans laquelle elle expose notamment son choix de se tourner vers le sionisme.

Le titre du recueil In land... fun mayn gebeyn nous invite en premier lieu à nous demander, sous la houlette de Kathryn Hellerstein, quel peut être ce pays : son pays natal ? son propre corps ? le pays où elle sera enterrée ? ${ }^{42}$ Il s'agit d'un recueil très lyrique (notamment si on le compare à sa production antérieure), une poésie saturée de symboles, très intimiste, dont le ressort est l'effort fait par la poétesse de transmuer à l'intérieur d'elle-même ce qui, désormais, est son exil. Les travaux et le travail de traduction passionnants effectués par K. Hellerstein mettent en évidence les caractéristiques essentielles de ce recueil qui n'est pas homogène. K. Hellerstein décrit finement comment le traitement de l'exil par Kadia Molodowsky évolue au fil du recueil : si les premiers poèmes témoignent d'une approche qu'on pourra qualifier de narrative, on s'achemine vers un système dans lequel les éléments même du langage, autrement dit les mots et les lettres, sont devenus les signes fragmentés de l'exil du poète. Au point paroxystique de ce cheminement, les lettres qui s'inscrivent sur le papier finissent par être perçues comme des îlots de stabilité perdus dans l'instabilité de l'exil. Le style narratif allégorique censé rendre compte de l'exil de la poétesse dans la première partie cède le pas à des poèmes assemblés tels des collages à partir de souvenirs fragmentés et de fragments de langue, tout ce qui reste d'elle à l'issue de ce processus d'atomisation.

$\mathrm{Au}$ détour du poème qui prête son titre au recueil, une maisonnette de shtetl, symbolisant la douceur maternelle, converse dans un dialogue surréaliste avec l'Empire State Building, incarnant la puissance masculine, quelques vers après que la poétesse a souligné le caractère intenable de la situation, cet entre-deux linguistique dont elle est prisonnière : «À cela, le yankee aurait répondu : O.K / Le litvak, lui, dit : tout juste bon à servir de volaille expiatoire avant Yom Kippour $\gg{ }^{43}$. La poétesse est littéralement coincée entre deux expressions idiomatiques intraduisibles qui incarnent à elles seules deux réponses culturelles à l'adversité, irréconciliables tant elles contiennent de manière exemplaire deux attitudes au monde différentes.

\footnotetext{
${ }^{42} \mathrm{~K}$. Hellerstein, « In Exile in the mother tongue », op. cit., p. 72.

${ }^{43} \mathrm{~K}$. Molodowski, In land ... fun mayn gebeyn, p. 46-47. Voir également l'analyse de K. Hellerstein, op. cit., p. 76. « Der yankee volt af dem gezogt : o-key / Der litvak zogt : es toygt af a kapore ».
} 
Dans le poème «Oysyes » ${ }^{44}$ [Lettres, au sens des lettres de l'alphabet], ce ne sont plus des mots, mais les lettres de l'alphabet qui deviennent le symbole de la condition exilique de la poétesse. Contrairement aux membres de sa famille installés outre-Atlantique, celle-ci est incapable de dire quelle est sa place en Amérique. Le poème dévoile le désarroi de la narratrice face à ces boutiquiers au bord de la faillite qui ont donné à leurs enfants des prénoms américains, sa déroute émotionnelle face à ces petits-cousins et petites-cousines arborant sur leurs chemises le nom de leur club de belote ou de leur compagnie de scouts brodé en lettres anglaises. Il dit aussi sa détresse devant l'enseigne en yiddish de la boutique familiale, laquelle renvoie à la poétesse son patronyme amputé dont elle égrène les premières lettres au fil des vers avant de renoncer. K. Molodowsky ne parvient pas à réconcilier les deux cultures dans sa poésie, elle est comme déchue de sa vie, de son écriture, de sa propre langue.

Le poème «Yerushe » $[\text { Héritage }]^{45}$, qui clôt l'un des cycles du recueil, constitue le point paroxystique de cette évolution : le doute, la perte de confiance en soi instillés par l'exil ont définitivement miné le système sémiotique ; tout ce qui reste, ce sont les lettres, mais des lettres dépourvues de signification, réduites à la qualité d'héritage, un héritage vide de sens. Malgré tout, ces lettres continuent d'exister comme des entités intactes, contrairement au Moi divisé, voire amorphe, du narrateur : "Seules les lettres sont transmises en héritage en abondance/ Léguées par le vieux melamed / Leyzer Ber avec sa pipe » ${ }^{46}$. Un autre élément significatif de ce poème est l'évocation - pour ne pas dire l'invocation - du vieux melamed, l'instituteur du système scolaire traditionnel, Leyzer Ber avec sa pipe: les personnages féminins qui étaient au cœur de la poésie de Kadya Molodowski, tout comme le souvenir de sa propre activité d'institutrice, cèdent donc la place à l'une des figures masculines par excellence de la culture traditionnelle. En cherchant une réponse dans la tradition patriarcale, Kadia Molodowsky semble verbaliser son propre échec de poétesse tournée vers la modernité.

\footnotetext{
${ }^{44}$ Ibid, p. 75.et K. Hellerstein, op. cit., p. 77-78.

${ }^{45} \mathrm{Ibid}$, p. 82. Pour l'analyse approfondie de K. Hellerstein, voir p. 79-80.

46 « Nor di oysyes zaynen geyarshnt voylgibik / Fun dem altn melamed / Leyzer Ber mitn tsibek ».
} 


\section{Conclusion}

En 1920 déjà, un des contributeurs de la revue Inzikh interrogeait : « La littérature yiddish a-t-elle un lectorat? La seule chose qui soit sûre, c'est qu'elle dispose d'écrivains ${ }^{47}$. De fait, la littérature yiddish aux États-Unis est doublement menacée: par la pauvreté du lectorat yiddishophone qui ne peut s'offrir le luxe de constituer une bibliothèque, puis par son ascension sociale, la réussite des immigrés Juifs les amenant à se détourner de cette littérature.

C'est ainsi que parvenus à leur maturité, les poètes du mouvement Di Yunge comprennent qu'ils appartiennent à nulle part et leur poésie retrouve les accents d'une poésie d'exil. Au fur et à mesure que le yiddish, en Amérique, devient une langue d'exil ${ }^{48}$, face à l'amenuisement du lectorat yiddishophone, face à l'afflux aussi de réfugiés et d'exilés au rythme des catastrophes qui frappent l'Europe et qui renvoient aux Juifs américains un autre spectre de l'identité juive, les plus grands des poètes prennent de plus en plus le chemin de l'introspection, trouvant en euxmêmes des ressources insoupçonnées.

Les introspectionnistes parviennent à une incandescence formelle en produisant une poésie qui est aussi profondément américaine et peut de fait entrer dans le canon de la littérature d'outre-Atlantique ${ }^{49}$. Les nuages qui s'amoncellent en Europe puis les premières nouvelles de l'extermination des Juifs d'Europe offrent aux écrivains yiddish américains l'occasion d'une véritable rencontre avec l'exil, qui se superpose à ce que furent leurs émotions fugaces au moment de leur venue, tous ayant fait, on ne saurait le nier, l'expérience du déplacement, de la perte du foyer ${ }^{50}$. Ce n'est plus la guerre ou des contraintes

\footnotetext{
47 In zikh, numéro de juin 1920. Cité dans la section «Chronicle of a movement Excerpts from Introspectivist Criticism » tirée de l'appendice de l'anthologie bilingue de poésie yiddish américaine de Barbara et Benjamin Harshav. Voir Barbara and Benjamin Harshav, American Yiddish Poetry - A Bilingual Anthology, Berkeley and Los Angeles, University of California Press, 1986, p. 791. La courte critique manifestement anonyme s'intitule « Ce cher lecteur».

${ }^{48}$ Comme le remarque judicieusement D.G. Roskies au détour de son essai sur l'Amérique dans l'espace imaginaire yiddish. Cf. David G. Roskies, « Coney Island, USA... ", op. cit., p. 83.

${ }^{49} \mathrm{Au}$ rebours par exemple d'un Moyshe Kulbak: sa période berlinoise est particulièrement prolifique, mais sa poésie ne parle jamais de Berlin, elle est tout sauf une poésie de la ville (bizarrement, c'est une fois installé à Minsk qu'il écrit sur Berlin, à savoir son célèbre poème Disner Tshayld Harold, 1928-1931).

${ }^{50}$ Voir par exemple Anita Norich, Discovering Exile: Yiddish and Jewish American Culture during the Holocaust, Stanford, Stanford University Press, 2007.
} 
juridiques qui désormais empêchent le retour, mais la destruction physique et irrémédiable de ce qui, à tout moment, était susceptible de constituer à nouveau un point d'attache. Ils ne sont plus aliénés du foyer, mais font face à une séparation radicale et irrévocable qui va conférer à leur poésie une dimension "chimiquement pure ${ }^{51}$. En cela, dans sa fêlure incurable, Kadia Molodowsky, anticipe cette expérience.

${ }^{51}$ C. Ksiazenicer-Matheron, « Dans la pliure du temps... », op. cit.. 